\title{
Probing turbulence with seismic observations of $\alpha$ Centauri
}

\author{
C. W. Straka ${ }^{1}$, P. Demarque ${ }^{1}$ and F. J. Robinson ${ }^{1,2}$ \\ ${ }^{1}$ Department of Astronomy, Yale University, New Haven, CT 06520-8101, USA \\ email: straka@astro.yale.edu, pierre.demarque@yale.edu \\ ${ }^{2}$ Department of Geology and Geophysics, Yale University, New Haven, CT 06520-8101, USA \\ email: marj@astro.yale.edu
}

\begin{abstract}
We present two 3D Large Eddy Simulations (LES) of the convection-radiation transition layer for both the A and the B component of the $\alpha$ Centauri binary system. Theoretical oscillation spectra are then calculated from stellar evolutionary models that include the effects of turbulence based on the LES simulations. By taking both non-seismic and seismic observational constraints into account we discuss the possibility to probe turbulence beyond standard mixing-length-theory in a star other than Sun.
\end{abstract}

Keywords. Turbulence, methods: numerical, stars: individual ( $\alpha$ Cen A, B), stars: oscillations

\section{Introduction}

Detailed three-dimensional hydrodynamic models of the outer convection-radiation transition layer in the Sun (Stein \& Nordlund 1998, Robinson et al. 2003) have shown that the superadiabatic stratification is not reproduced with mixing-length theory (MLT, Böhm-Vitense 1958), which is still widely used in stellar modeling. New observational techniques to measure $p$-mode oscillation frequencies from the ground (Kjeldsen et al. 1995, Martić et al. 1999) and their subsequent refinement have led to a number of unambiguous detections of global oscillations of solar-type stars (e.g., Bedding \& Kjeldsen 2003). These observations are now making it worthwhile to consider turbulence effects on solar-type stars other than the Sun.

A first attempt in this direction was made by Straka et al. (2006) who explored the structural changes of the outer layers of $\eta$ Boo due to turbulence as well as the subsequent effects on the theoretical p-mode frequencies. While this study showed that turbulence imposes a measurable effect on the p-mode frequencies in the frequency range of the ground-based observations, the results were still based on a solar LES. One of the two most intensively studied solar-type stars other than Sun are $\alpha$ Centauri ( $\alpha$ Cen) A and B. New ground-based observations of p-mode oscillations on both the A and (Bouchy \& Carrier 2002, Bedding et al. 2004) and the B component (Carrier \& Bourban 2003, Kjeldsen et al. 2005) provide a unique opportunity for investigating the role turbulence plays in their outer stellar layers. In this study, we calculate theoretical model frequencies that take turbulence effects into account and compare them to the observations.

\section{Results from 3D large eddy simulations}

We performed two 3D large eddy simulations (LES) each in a box with $97 \times 97 \times 180$ grid points, one for $\alpha$ Cen A and one for $\alpha$ Cen B. We are using the Kim \& Chan (1998) LES code and a geometric setup which slightly exceeds the ranges found necessary for achieving realistic flow patterns in the Sun (Robinson et al. 2003). 

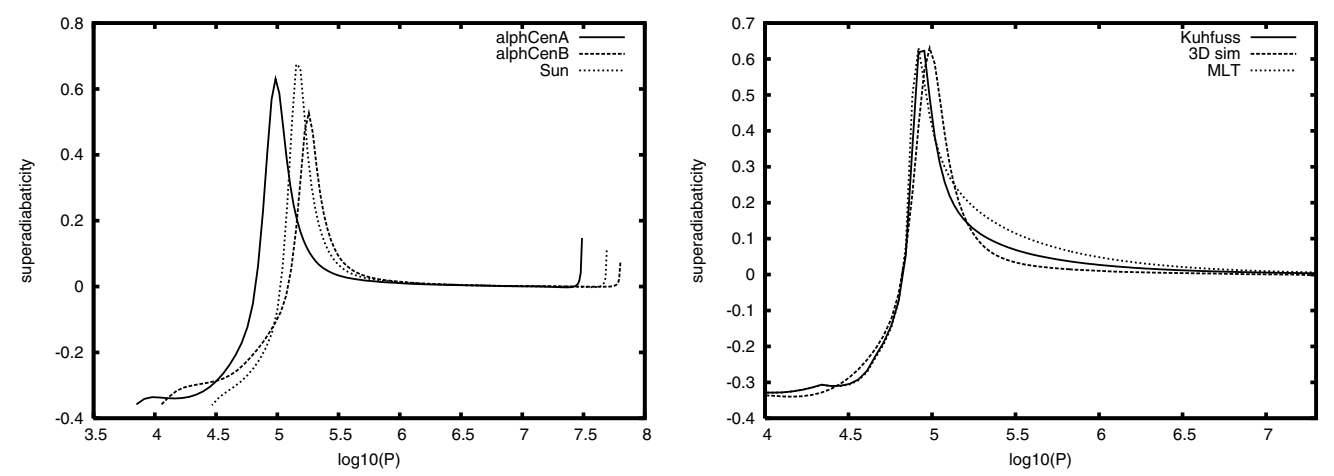

Figure 1. Superadiabatic layer for $\alpha$ Cen as calculated from 3D LES. (a) Compared to solar LES. (b) Compared to simple one-dimensional convection theories (only $\alpha$ Cen A shown).

As outlined in Robinson et al. (2003) the fully compressible Navier-Stokes equations are solved and the radiative transfer is treated self-consistently with the hydrodynamics under the assumption that retardation-effects can be neglected. In the optically thin region up to optical depths $\tau \sim 10^{4}$ the mean intensity $J$ is computed using the generalized three-dimensional Eddington approximation (Unno \& Spiegel 1966), allowing non-equilibrium conditions between the gas and the radiation temperature. The computational efficiency and stability of the radiative transfer solver has been improved with the multigrid method (Straka \& Demarque 2006).

\subsection{LES simulations for $\alpha$ Cen $A$ and $B$}

After the simulations had reached a relaxed state, statistics were accumulated over more than 60 eddy turnover times. We checked for statistical convergence in second-order turbulent quantities, such as the horizontal Reynolds stress. Figure 1a depicts the superadiabatic layer (SAL) of both stars in comparison to the SAL of the Sun. The location of the solar SAL peak is bracketed by the $\alpha$ Cen A and B components as expected. In Figure 1b the structure from the LES is compared with the structure predicted by 1-dimensional convection theories. The free mixing length parameter was calibrated to yield the same peak height. The largest discrepancy is found with MLT. The Kuhfuss 1986 convection theory is an improvement, most notably in the sub-photospheric layers. Adjusting the additional free parameters does not improve the fit.

\subsection{Influence on $p$-mode oscillations}

The changes to the structure of the outer layers of $\alpha$ Cen A and B based on the LES turbulent kinetic energy and turbulent pressure are calculated with the methods described by Li et al. (2002). The structural changes due to turbulence lead to differences in the p-mode oscillation frequencies and are shown for $\alpha$ Cen A (Figure 2a) and for $\alpha$ Cen $\mathrm{B}$ (Figure 2b). The frequency range plotted was chosen to coincide with the range of observed modes. The frequency ridge in these echelle diagrams shifts by approximately $5 \mu \mathrm{Hz}$ for $\alpha$ Cen A and $10 \mu \mathrm{Hz}$ for $\alpha$ Cen B between a standard model and a model based on the LES. The differences are largest at the high frequency cutoff and diminish at the bottom of the frequency window.

\subsection{Influence on large separations}

The large separations are also affected (Figure 2c). The modifications due to turbulence when combined with those from the non-adiabaticity of the modes lead to a decrease of the averaged large separations by $1 \mu \mathrm{Hz}$. In Figure $2 \mathrm{~d}$ we show the large separations as 

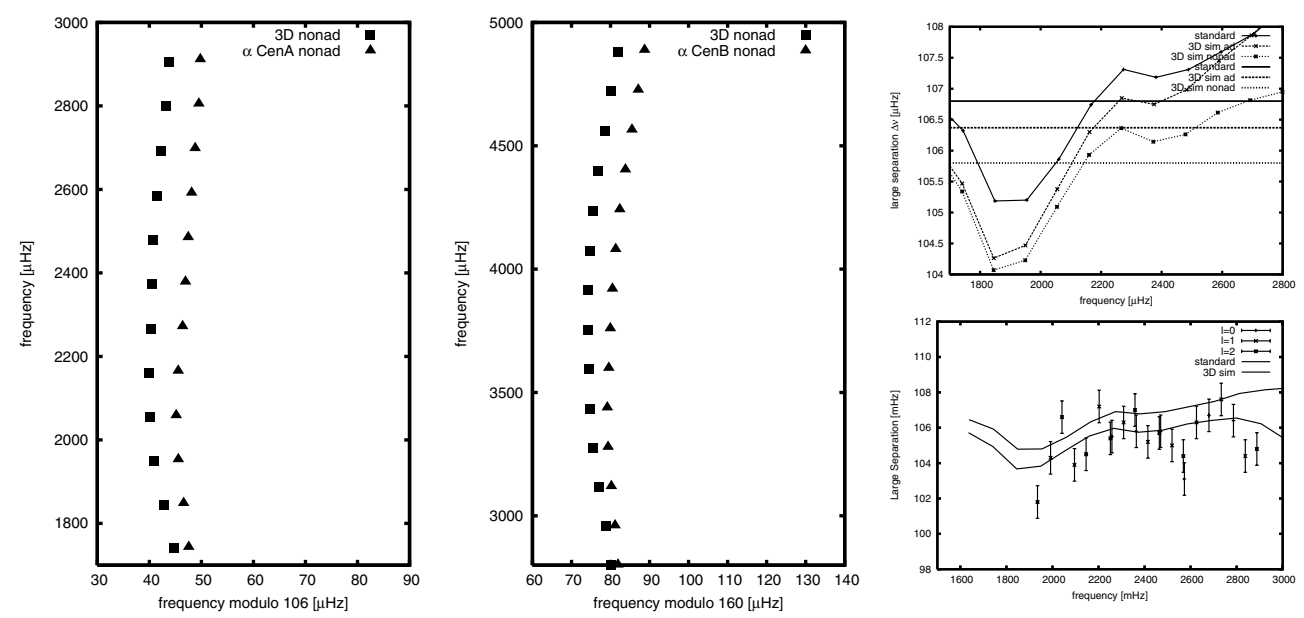

Figure 2. Theoretical echelle diagrams for models with and without turbulence for (a) $\alpha$ Cen A and (b) $\alpha$ Cen B. (c) Corresponding effects on (averaged) large separations . (d) Comparison with observational data and model predictions from Miglio \& Montalbán (2005).

given by the observational data from Bouchy \& Carrier (2002) with $2 \sigma$ error bars. The upper line is the prediction of the large separations from the stellar models by Miglio \& Montalbán (2005) who take both classical and seismic constraints into account. As noted by the these authors, their models predict a large separation that is about $1 \mu \mathrm{Hz}$ too high in comparison to the observations, if the independent interferometric radius measurement (Kervella et al. 2003) is used as a constraint. This discrepancy can be understood in terms of the results presented here: the structural changes due to turbulence lead to a decrease of the large separations by $1 \mu \mathrm{Hz}$.

\section{Acknowledgements}

This research is supported in part by NASA grant NAG5-13299.

\section{References}

Bedding, T. R. \& Kjeldsen, H. 2003, Pub. Astron. Soc. Aus., 20, 203

Bedding, T. R., Kjeldsen, H., Butler, R. P., McCarthy, C., et al. 2004, Astrophys. J., 614, 380

Böhm-Vitense, E. 1958, Zeitschrift für Astrophysik, 46, 108

Bouchy, F. \& Carrier, F. 2002, Aastron. \& Astrophys., 390, 205

Carrier, F. \& Bourban, G. 2003, Aastron. Es Astrophys., 406, L23

Kervella, P., Thévenin, F., Ségransan, D., et al. 2003, Aastron. ES Astrophys., 404, 1087

Kim, Y.-C. \& Chan, K.L. 1998, Astrophys. J., 496, L121

Kjeldsen, H., Bedding, T. R., Viskum, M. \& Frandsen, S. 1995, Astron. J., 109, 1313

Kjeldsen, H., et al. 2005, Astrophys. J., 635, 1281

Kuhfuss, R. 1986, Aastron. \& Astrophys., 160, 116

Li, L. H., Robinson, F. J., Demarque, P., Sofia, S., et al. 2002, Astrophys. J., 567, 1192

Martić, M., et al. 1999, Aastron. \& Astrophys., 351, 993

Miglio, A. \& Montalbán, J. 2005, Aastron. \& Astrophys., 441, 615

Robinson, F. J., Demarque, P., Li, L. H., et al. 2003, Mon. Not. Roy. Astron. Soc., 340, 923

Stein, R. F. \& Nordlund, A. 1998, Astrophys. J., 499, 914

Straka, C. W., Demarque, P., Guenther, D. B., Li, L., et al. 2006, Astrophys. J., 636, 1078

Straka, C. W. \& Demarque, P. 2006, Comp. Phys. Comm., submitted

Unno, W. \& Spiegel, E. A. 1966, Publ. Aston. Soc. Japan, 18, 85 